\title{
COLLABORATIVE EXPLORATION IN GRID DOMAINS Constructive Conjecture of a Polynomial Time Complexity
}

\author{
Yaniv Altshuler \\ Deutsche Telekom Laboratories, Ben Gurion University, Beer Sheva, Israel \\ yanival@cs.technion.ac.il \\ Alfred M. Bruckstein \\ Israel Institute of Technology, Computer Science Department, Technion, Technion City, Haifa 32000, Israel \\ freddy@cs.technion.ac.il \\ Israel A. Wagner \\ IBM Haifa Research Labs, Haifa, Israel \\ wagner@il.ibm.com
}

Keywords: Swarm Algorithm, Decentralized Search, Cooperative Exploration, Grid Exploration, Time Complexity

Abstract: $\quad$ This work discusses the problem of exploration of an unknown environment using a collaborative group of simple agents. While this problem was known to be of a non-polynomial time complexity, it was speculated in the past that in grid domains the completion time of this problem is much lower (although analytic proofs were not available hitherto). In this work we present a preliminary result concerning a constructive analytic constraint for guaranteeing that the time complexity of this problem in grid domains is indeed polynomial.

\section{INTRODUCTION}

In recent years significant research efforts have been invested in design and simulation of multiagent robotics and intelligent swarms systems see e.g. (Hettiarachchi and Spears, 2005; Wagner and Bruckstein, 2001; Steels, 1990) or (Arkin, 1990; Mataric, 1992; Haynes and Sen, 1986) for biology inspired designs (behavior based control models, flocking and dispersing models and predator-prey approaches, respectively), (Gerkey and Mataric, 2002; Rabideau et al., 1999; Thayer et al., 2000) for economics applications and (Chevallier and Payandeh, 2000) for a physics inspired approach).

Tasks that have been of particular interest to researchers in recent years include synergetic mission planning (Alami et al., 1998), swarm control (Mataric, 1994), human design of mission plans (MacKenzie et al., 1997), role assignment (Candea et al., 2001), multi-robot path planning (Yamashita et al., 2000), formation generation (Gordon et al., 2003), formation keeping (Balch and Arkin, 1998), exploration and mapping (Rekleitis et al., 2003), cleaning (Wagner et al., 2008) target tracking (Shucker and Bennett, 2005) and many more.

Unfortunately, the mathematical \geometrical theory of such multi-agents systems is far from being satisfactory, as pointed out in (Beni and Wang, 1991; E.Bonabeau et al., 1999; Efraim and D.Peleg, 2007;
Olfati-Saber, 2006) and many other papers.

One of the most interesting challenges for a robotics swarm system is the design and analysis of a multi-robotics system for searching and exploration (in either known or unknown areas). For example, works discussing cooperative searching tasks for static or dynamic targets can be found in (Altshuler et al., 2005b; Kerr and Spears, 2005; Passino et al., 2002; Polycarpou et al., 2001; Stone, 1975; Koopman, ; Vincent and Rubin, 2004) whereas examples for cooperative coverage of given regions are presented in (Rekleitisy et al., 2004; Rekleitis et al., 2005; Kong et al., 2006).

In this work we discuss the general problem of collaborative search of an unknown grid domains by a decentralized group of ant-like robots, while trying to establish an analytic proof for its time complexity. The strategy that will be used will based on the presentation of an "archetype problem", to which an analytic upper bound over its completion time will be presented. From this bound, the time complexity of the problem will be derived, and shown to be polynomial (when the bound predicts that the problem can be solved). Cases where the bound does not predict a successful completion of the mission are not treated here, although it is our belief that further analysis of the bound may yield a similar result for these cases as well.

Existing approaches and results are discussed in 
Section 2, while a conjecture concerning the time complexity in grid domains is presented in Section 3.

\section{RELATED WORK AND MOTIVATION}

In general, most of the techniques used for the task of a distributed coverage use some sort of cellular decomposition. For example, in (Rekleitisy et al., 2004) the area to be covered is divided between the agents based on their relative locations. In (Butler et al., 2001) a different decomposition method is being used, which is analytically shown to guarantee a complete coverage of the area. Another interesting work is presented in (Acar et al., 2001), discussing two methods for cooperative coverage (one probabilistic and the other based on an exact cellular decomposition). All of the works mentioned above, however, rely on the assumption that the cellular decomposition of the area is possible. This in turn, requires the use of memory resources, used for storing the dynamic map generated, the boundaries of the cells, etc'. As the initial size and geometric features of the area are generally not assumed to be known in advance, agents equipped with merely a constant amount of memory will most likely not be able to use such algorithms.

Surprisingly, while existing works concerning distributed (and decentralized) coverage often present analytic proofs for the ability of the system to guarantee the completion of the task (for example, in (Acar et al., 2001; Butler et al., 2001; Batalin and Sukhatme, 2002)), unfortunately, most of them lack analytic bounds for the coverage time (although in many cases an extensive amount of empirical results of this nature is made available). It can well be said that the time complexity of this problem, as well as the similar results for a variety of its derivatives, are still unavailable.

An interesting work to mention in this scope is this of (Svennebring and Koenig, 2004; Koenig and Liu, 2001), where a swarm of ant-like robots is used for repeatedly covering an unknown area, using a real time search method called node counting. By using this method, the robots are shown to be able to efficiently perform such a coverage mission, and analytic bounds for the coverage time are discussed. Based on a more general result for undirected domains shown in (Koenig et al., 2001), the following bound is given :

The cover time of teams of ant robots (of a given size) that use node counting on strongly connected undirected graphs can be exponen- tial in the square root of the number of vertices.

Namely :

$$
f(k)=O\left(2^{\sqrt{S_{0}}}\right)
$$

denoting the covering time of $k$ robots by $f(k)$, and the initial area of the region to be explored by $S_{0}$.

It should be mentioned though, that in (Svennebring and Koenig, 2004) the authors clearly state that it is their belief that the coverage time of robots using nodes counting in grids is much smaller. This estimation is also demonstrated experimentally. However, no analytic evidence for this was available thus far. As grid domains are often used as an approximation for a problem in $\mathbf{R}^{2}$, the importance of such result is also likely to supersede its relevance to "purely discrete" problems.

\section{TIME COMPLEXITY IN GRID DOMAINS}

For dealing with the generic problem of exploring an unknown grid domain, we propose to discuss the "Dynamic Cooperative Cleaners" problem, a problem that requires several simple agents to clean a connected region of "dirty" pixels in $\mathbf{Z}^{2}$. A number of simple agents move in this dirty region, unaware of its size of shape, each having the ability to "clean" the place it is located in. Their goal being to jointly clean the given dirty region. The dynamic generalization of the problem involves a deterministic expansion of dirt in the environment, simulating spreading of contamination, or fire. By controlling the initial "contaminated" region any kind of search space in $\mathbf{Z}^{2}$ can be simulated (and any search space in $\mathbf{R}^{2}$ can be approximated). By employing the dynamic property of the contamination, other known problems can be derived. For example, collaborative hunting for evading targets (Altshuler et al., 2005b; Vincent and Rubin, 2004) may be simulated by using spreading contamination as the union of all possible target's locations.

It is also important to note that as the agents used in this problem are assumed to be as limited as possible (see more details in Section 3.1, any result obtained for this model will automatically be applicable for almost any other model of agents conceivable.

\subsection{Cooperative Cleaners Problem}

We shall assume that the time is discrete. Let the undirected graph $G(V, E)$ denote a two dimensional integer grid $\mathbf{Z}^{2}$, whose vertices (or "tiles") have a binary property called 'contamination'. Let $\operatorname{cont}_{t}(v)$ 
state the contamination state of the tile $v$ at time $t$, taking either the value "on" or "off". Let $F_{t}$ be the contaminated sub-graph of $G$ at time $t$, i.e. : $F_{t}=$ $\left\{v \in G \mid \operatorname{cont}_{t}(v)=\right.$ on $\}$. We assume that $F_{0}$ is a single connected component. Our algorithm will preserve this property along its evolution.

Let a group of $k$ agents that can move on the grid $G$ (moving from a tile to its neighbor in one time step) be placed at time $t_{0}$ on $F_{0}$, at point $p_{0} \in F_{t}$. Each agent is equipped with a sensor capable of telling the contamination status of all tiles in the digital sphere of diameter 7, which surrounds the agent. An agent is also aware of other agents which are located in these tiles, and all the agents agree on a common direction. Each tile may contain any number of agents simultaneously. Each agent is equipped with a memory of size $O(\log k)$ bits. When an agent moves to a tile $v$, it has the possibility of cleaning this tile (i.e. causing $\operatorname{cont}(v)$ to become off. The agents do not have any prior knowledge of the shape or size of the sub-graph $F_{0}$ except that it is a single and simply connected component.

The contaminated region $F_{t}$ is assumed to be coated at its boundary by a rubber-like elastic barrier, dynamically reshaping itself to fit the evolution of the contaminated region over time. This barrier is intended to guarantee the preservation of the simple connectivity of $F_{t}$, crucial for the operation of the agents, due to their limited memory. When an agent cleans a contaminated tile, the barrier retreats, in order to fit the void previously occupied by the cleaned tile. Every $d$ time steps the contamination spreads. That is, if $t=n d$ for some positive integer $n$, then :

$$
\forall v \in F_{t} \forall u \in 4-\operatorname{Neighbors}(v), \operatorname{cont}_{t+1}(u)=\text { on }
$$

While the contamination spreads, the elastic barrier stretches while preserving the simple-connectivity of the region, as demonstrated in Figure 1. For the agents who travel along the tiles of $F$, the barrier signals the boundary of the contaminated region.

The agents' goal is to clean $G$ by eliminating the contamination entirely, meaning that : $\left(\exists t_{\text {success }}\right.$ s.t $\left.F_{t_{\text {success }}}=\emptyset\right)$. In addition, it is desired that time $t_{\text {success }}$ will be minimal.

It is important to note that no central control is allowed, and that the system is fully decentralized (i.e. all agents are identical and no explicit communication between the agents is allowed). An important advantage of this approach, in addition to the simplicity of the agents, is fault-tolerance - even if almost all the agents cease to work before completion, the remaining ones will eventually complete the mission, if possible.

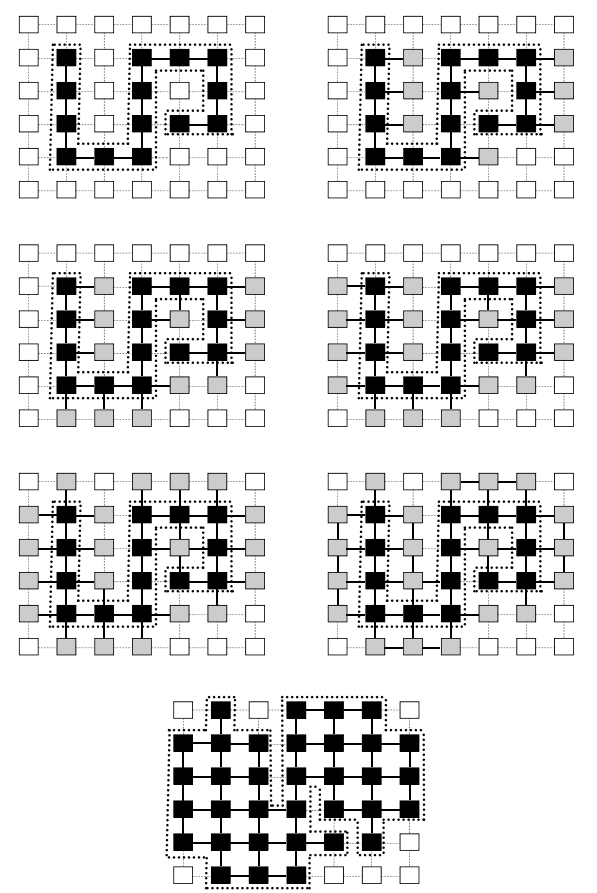

Figure 1: A demonstration of the barrier expansion process as a result of a contamination spread.

\subsection{Existing Results}

The cooperative cleaners problem was previously studied in (Wagner et al., 2008) (static version) and (Altshuler et al., 2005a; Altshuler et al., 2009) and others (dynamic version). A cleaning algorithm was proposed (used by a decentralized group of simple mobile agents, for exploring and cleaning an unknown "contaminated" sub-grid $F$, expanding every $d$ time steps) and its performance analyzed. Following are two existing results containing upper bounds on the cleaning algorithm's completion time.

For some values of $d$ and $k$, the cleaning might be completed in $t_{\text {static }}$ time steps, even before the contamination is able to spread even once:

Theorem 1 If $t_{\text {static }}<d$, then the region is known to be cleaned in less than d time steps (namely, before any contamination spread), where :

$$
t_{\text {static }} \triangleq \frac{8\left(\left|\partial F_{0}\right|-1\right) \cdot\left(W\left(F_{0}\right)+k\right)}{k}+2 k
$$

where $W(F)$ is the region's depth (the shortest path from some internal point in $F$ to its surface, for the internal point whose said shortest path is the longest) and $\partial F$ denotes the boundary of $F$, defined as :

$\partial F=\{v \mid v \in F \wedge 8-\operatorname{Neighbors}(v) \cap(G \backslash F) \neq \emptyset\}$ 
For a contaminated region $F_{0}$, for which the contamination is predicted to spread before being cleaned, then the following result is available :

Theorem 2 Let $\mu_{\text {success }} \triangleq \min \left\{x \in\left\{\mu_{1}, \mu_{2}\right\} \mid x>0\right\}$ where $\mu_{1}$ and $\mu_{2}$ are defined as :

$\frac{\left(A_{4}-A_{1} A_{3}\right) \pm \sqrt{\left(A_{1} A_{3}-A_{4}\right)^{2}-4 A_{3}\left(A_{2}-A_{1}-A_{1} A_{4}\right)}}{2 A_{3}}$

where :

$$
\begin{gathered}
A_{1}=\frac{c_{0}+2-\gamma_{2}}{4}, A_{2}=\frac{c_{0}+2+\gamma_{2}}{4}, A_{3}=\frac{8 \cdot \gamma_{2}}{d \cdot k} \\
A_{4}=\gamma_{1}-\frac{\gamma_{2} \cdot \gamma}{d}, \gamma_{1}=\psi\left(1+A_{2}\right)-\psi\left(1+A_{1}\right) \\
\gamma_{2}=\sqrt{\left.\left(c_{0}+2\right)^{2}-8 S_{0}+8\right)} \\
\gamma=\frac{8\left(k+W\left(F_{0}\right)\right)}{k}-\frac{d-2 k}{\left|\partial F_{0}\right|-1}
\end{gathered}
$$

If such $\mu_{\text {SUCCESS }}$ exists then $t_{\text {success }}=\left(\mu_{\text {success }}-1\right) \cdot d$.

Where $c_{0}$ is the circumference of the initial region $F_{0}$, and where $\psi()$ is the Digamma function (studied in (Abramowitz and Stegun, 1964)) — the logarithmic derivative of the Gamma function, defined as :

$$
\psi(x)=\frac{d}{d x} \ln \Gamma(x)=\frac{\Gamma^{\prime}(x)}{\Gamma(x)}
$$

or as :

$$
\psi(x)=\int_{0}^{\infty}\left(\frac{e^{-t}}{t}-\frac{e^{-x t}}{1-e^{-t}}\right) d t
$$

\subsection{Grid Exploration Complexity}

Using Theorem1, as $\left|\partial F_{0}\right|=O\left(S_{0}\right)$ and as $W\left(F_{0}\right)=$ $O\left(\sqrt{S_{0}}\right)$ we see that :

$$
t_{\text {static }}(k)=O\left(\frac{1}{k} S_{0}^{1.5}+S_{0}+k\right)
$$

As for practical reasons we assume that $k<S_{0}$ we can see that :

$$
t_{\text {static }}(k)=O\left(\frac{1}{k} S_{0}^{1.5}+S_{0}\right)
$$

and when the number of robots is independent in the size of the region, we can write :

\section{Corollary 1 If}

$$
\frac{8\left(\left|\partial F_{0}\right|-1\right) \cdot\left(W\left(F_{0}\right)+k\right)}{k}+2 k<d
$$

Then

$$
t_{\text {static }}(k)=O\left(S_{0}^{1.5}\right)
$$

The analysis becomes slightly more complicated when $\frac{8\left(\left|\partial F_{0}\right|-1\right) \cdot\left(W\left(F_{0}\right)+k\right)}{k}+2 k \geq d$, namely - when the contamination spreads before the robots had completed its cleaning. In this case, the cleaning time of the robots might be significantly longer, as clean tiles might become contaminated once again, while the robots are busy cleaning the evolving contaminated region. In order to analyze the complexity of the robots' cleaning time we shall use Theorem 2 :

Therefore :

$t_{S U C C E S S}=$

$$
\begin{gathered}
d \cdot O\left(A_{1}+\frac{A_{4}}{A_{3}}+\sqrt{A_{1}^{2}+\frac{A_{1} A_{4}+A_{1}+A_{2}}{A_{3}}+\frac{A_{4}^{2}}{A_{3}^{2}}}\right)= \\
d \cdot O\left(A_{1}+\frac{A_{4}}{A_{3}}+A_{1}+\frac{\sqrt{A_{3}} \sqrt{A_{1} A_{4}+A_{1}+A_{2}}}{A_{3}}+\frac{A_{4}}{A_{3}}\right)= \\
d \cdot O\left(A_{1}+\frac{A_{4}}{A_{3}}+\frac{\sqrt{A_{1} A_{4}}+\sqrt{A_{1}}+\sqrt{A_{2}}}{\sqrt{A_{3}}}\right)= \\
d \cdot O\left(c_{0}+\gamma_{2}+d k \frac{\gamma_{1}}{\gamma_{2}}+k \gamma+\sqrt{k} \sqrt{c_{0}+\gamma_{2}} \sqrt{\gamma}\right)+ \\
+d \cdot O\left(\sqrt{k} \frac{\sqrt{c_{0}+\gamma_{2}}\left(\sqrt{d \gamma_{1}}+1\right)}{\sqrt{\gamma_{2}}}\right)
\end{gathered}
$$

Taking into consideration that $\left(\gamma_{2} \in \mathbb{R}\right) \Rightarrow\left(\gamma_{2} \geq 1\right)$ and that $\gamma_{2}=O\left(c_{0}\right)$ we can write the previous expression as :

$$
d \cdot O\left(c_{0}+d k \gamma_{1}+k \gamma+\sqrt{k} \sqrt{c_{0}}\left(\sqrt{d \gamma_{1}}+1+\sqrt{\gamma}\right)\right)
$$

As $\left|\partial F_{0}\right|=O\left(S_{0}\right)$ and as $W\left(F_{0}\right)=O\left(\sqrt{S_{0}}\right)$ we can see that :

$$
\gamma=O\left(\frac{\sqrt{S_{0}}}{k}+\frac{d+k}{\sqrt{S_{0}}}\right)
$$

and when we require that the number of robots is independent of the size of the region, we can assume that $k<\sqrt{S_{0}}$ and write :

$$
\gamma=O\left(\sqrt{S_{0}}+\frac{d}{\sqrt{S_{0}}}\right)
$$

And as the delay between two spreads can assumed to be smaller than the size of the region, we can see that $\gamma=O\left(\sqrt{S_{0}}\right)$. In addition, remembering that $O\left(\sqrt{S_{0}}\right) \leq c_{0} \leq O\left(S_{0}\right)$ we can rewrite the expression of equation 2 as follows :

$$
t_{\text {SUCCESS }}=d \cdot O\left(c_{0}+d k \gamma_{1}+k \sqrt{S_{0}}+\sqrt{k d c_{0} \gamma_{1}}+\sqrt{k} S_{0}\right)
$$

Let us observe $\gamma_{1}$ :

$$
\gamma_{1} \triangleq \psi\left(1+\frac{c_{0}+2+\gamma_{2}}{4}\right)-\psi\left(1+\frac{c_{0}+2-\gamma_{2}}{4}\right)
$$


As $\left(\gamma_{2} \in \mathbb{R}\right) \Rightarrow\left(\gamma_{2}>0\right)$ we are guaranteed that $\left(1+\frac{c_{0}+2+\gamma_{2}}{4}\right)>1$. Note that $\psi(1)=-\hat{\gamma}$ where $\hat{\gamma}$ is the Euler-Mascheroni constant, defined as :

$\hat{\gamma}=\lim _{n \rightarrow \infty}\left[\left(\sum_{k=1}^{n} \frac{1}{k}\right)-\log (n)\right]=\int_{1}^{\infty}\left(\frac{1}{\lfloor x\rfloor}-\frac{1}{x}\right) d x$

which equals approximately 0.57721 . In addition, $\psi(x)$ is monotonically increasing for every $x>0$. As we also know that $\psi(x)$ is upper bounded by $O(\ln (x))$ for large values of $x$, we see that :

$$
-0.58<\psi\left(1+\frac{c_{0}+2+\gamma_{2}}{4}\right)<O\left(\ln \left(c_{0}\right)\right)
$$

We now need to present a bound for $\psi\left(1+\frac{c_{0}+2-\gamma_{2}}{4}\right)$. For this, it is sufficient to show that $\left(1+\frac{c_{0}+2-\gamma_{2}}{4}\right)>1$. It is easy to see that this holds when $c_{0} \geq \gamma_{2}$ :

$$
\begin{aligned}
& c_{0} \quad ? \geq \gamma_{2} \triangleq \sqrt{\left(c_{0}+2\right)^{2}-8 \cdot\left(S_{0}-1\right)} \quad \Longrightarrow \\
& c_{0}^{2} \quad ? \geq\left(c_{0}+2\right)^{2}-8 \cdot\left(S_{0}-1\right)=c_{0}^{2}+4 c_{0}-4-8 S_{0} \\
& 2 S_{0}+1 \quad ? \geq c_{0}
\end{aligned}
$$

which of course holds, as $c_{0} \leq 2 S_{0}-2$. Therefore $\left(1+\frac{c_{0}+2-\gamma_{2}}{4}\right)>1$ which implies :

$$
-0.58<\psi\left(1+\frac{c_{0}+2-\gamma_{2}}{4}\right)<O\left(\ln \left(c_{0}\right)\right)
$$

Combining equations 4 and 5 we see that :

$$
-O\left(\ln \left(c_{0}\right)\right)<\gamma_{1}<O\left(\ln \left(c_{0}\right)\right)
$$

Using equation 6 in order to rewrite equation 3 produces :

\section{Conjecture 1}

$t_{S U C C E S S}=$

$d \cdot O\left(c_{0}+d k \ln \left(c_{0}\right)+k \sqrt{S_{0}}+\sqrt{d k c_{0} \ln \left(c_{0}\right)}+\sqrt{k} S_{0}\right)$

Comparing this to the bound of equation 1 , we see that when successful completion of the mission by the cleaning protocol proposed is guaranteed, its time complexity is polynomial, as $c_{0}, d, k<O\left(S_{0}\right)$.

\section{Conclusions}

In this work the decentralized exploration problem using a group of simple, ant-like agents was discussed. A conjecture suggesting that the time complexity of this problem in grid domain is polynomial was presented. This was done by introducing a constraint which when followed guarantees a polynomial time completion. The importance of this result, as well as several related work were also discussed.

\section{REFERENCES}

Abramowitz, M. and Stegun, I. (1964). Handbook of Mathematical Functions, page 55. Applied Mathematics Series. National Bureau of Standards.

Acar, E., Zhang, Y., Choset, H., Schervish, M., Costa, A., Melamud, R., Lean, D., and Gravelin, A. (2001). Path planning for robotic demining and development of a test platform. In International Conference on Field and Service Robotics, pages 161-168.

Alami, R., Fleury, S., Herrb, M., Ingrand, F., and Robert, F. (1998). Multi-robot cooperation in the martha project. IEEE Robotics and Automation Magazine, 5(1):3647.

Altshuler, Y., Bruckstein, A., and Wagner, I. (2005a). Swarm robotics for a dynamic cleaning problem. In IEEE Swarm Intelligence Symposium, pages 209-216.

Altshuler, Y., Wagner, I., and Bruckstein, A. (2009). Swarm ant robotics for a dynamic cleaning problem - upper bounds. The 4th International conference on Autonomous Robots and Agents (ICARA-2009), pages 227-232.

Altshuler, Y., Yanovski, V., Wagner, I., and Bruckstein, A. (2005b). The cooperative hunters - efficient cooperative search for smart targets using uav swarms. In Second International Conference on Informatics in Control, Automation and Robotics (ICINCO), the First International Workshop on Multi-Agent Robotic Systems (MARS), pages 165-170.

Arkin, R. (1990). Integrating behavioral, perceptual, and world knowledge in reactive navigation. Robotics and Autonomous Systems, 6:105-122.

Balch, T. and Arkin, R. (1998). Behavior-based formation control for multi-robot teams. IEEE Transactions on Robotics and Automation, 14(6):926-939.

Batalin, M. and Sukhatme, G. (2002). Spreading out: A local approach to multi-robot coverage. In 6th International Symposium on Distributed Autonomous Robotics Systems.

Beni, G. and Wang, J. (1991). Theoretical problems for the realization of distributed robotic systems. In IEEE Internal Conference on Robotics and Automation, pages 1914-1920.

Butler, Z., Rizzi, A., and Hollis, R. (2001). Distributed coverage of rectilinear environments. In Proceedings of the Workshop on the Algorithmic Foundations of Robotics.

Candea, C., Hu, H., Iocchi, L., Nardi, D., and Piaggio, M. (2001). Coordinating in multi-agent robocup teams. Robotics and Autonomous Systems, 36(2-3):67-86.

Chevallier, D. and Payandeh, S. (2000). On kinematic geometry of multi-agent manipulating system based on the contact force information. In The Sixth International Conference on Intelligent Autonomous Systems (IAS-6), pages 188-195.

E.Bonabeau, M.Dorigo, and G.Theraulaz (1999). Swarm Intelligence: From Natural to Artificial Systems. Oxford University Press, US. 
Efraim, A. and D.Peleg (2007). Distributed algorithms for partitioning a swarm of autonomous mobile robots. Structural Information and Communication Complexity, Lecture Notes in Computer Science, 4474:180194.

Gerkey, B. and Mataric, M. (2002). Sold! market methods for multi-robot control. IEEE Transactions on Robotics and Automation, Special Issue on Multirobot Systems.

Gordon, N., Wagner, I., and Bruckstein, A. (2003). Discrete bee dance algorithms for pattern formation on a grid. In IEEE International Conference on Intelligent Agent Technology (IAT03), pages 545-549.

Haynes, T. and Sen, S. (1986). Evolving Behavioral Strategies in Predators and Prey, volume 1042 of Lecture Notes in Computer Science, chapter Adaptation and Learning in Multi-Agent Systems, pages 113-126. Springer, Berlin.

Hettiarachchi, S. and Spears, W. (2005). Moving swarm formations through obstacle fields. In International Conference on Artificial Intelligence.

Kerr, W. and Spears, D. (2005). Robotic simulation of gases for a surveillance task. In Intelligent Robots and Systems (IROS 2005), pages 2905-2910.

Koenig, S. and Liu, Y. (2001). Terrain coverage with ant robots: A simulation study. In AGENTS'01.

Koenig, S., Szymanski, B., and Liu, Y. (2001). Efficient and inefficient ant coverage methods. Annals of Mathematics and Artificial Intelligence, 31:41-76.

Kong, C., Peng, N., and Rekleitis, I. (2006). Distributed coverage with multi-robot system. In IEEE International Conference on Robotics and Automation.

Koopman, B. The theory of search ii, target detection. $O p$ erations Research.

MacKenzie, D., Arkin, R., and Cameron, J. (1997). Multiagent mission specification and execution. Autonomous Robots, 4(1):29-52.

Mataric, M. (1992). Designing emergent behaviors: From local interactions to collective intelligence. In J.Meyer, H.Roitblat, and S.Wilson, editors, Proceedings of the Second International Conference on Simulation of Adaptive Behavior, pages 432-441. MIT Press.

Mataric, M. (1994). Interaction and Intelligent Behavior. $\mathrm{PhD}$ thesis, Massachusetts Institute of Technology.

Olfati-Saber, R. (2006). Flocking for multi-agent dynamic systems: Algorithms and theory. IEEE Transactions on Automatic Control, 51(3):401-420.

Passino, K., Polycarpou, M., Jacques, D., Pachter, M., Liu, Y., Yang, Y., Flint, M., and Baum, M. (2002). Cooperative Control for Autonomous Air Vehicles, chapter Cooperative Control and Optimization. Kluwer Academic, Boston.

Polycarpou, M., Yang, Y., and Passino, K. (2001). A cooperative search framework for distributed agents. In IEEE International Symposium on Intelligent Control, pages $1-6$.
Rabideau, G., Estlin, T., Chien, T., and Barrett, A. (1999). A comparison of coordinated planning methods for cooperating rovers. In Proceedings of the American Institute of Aeronautics and Astronautics (AIAA) Space Technology Conference.

Rekleitis, I., Dudek, G., and Milios, E. (2003). Experiments in free-space triangulation using cooperative localization. In IEEE/RSJ/GI International Conference on Intelligent Robots and Systems (IROS).

Rekleitis, I., New, A., and Choset, H. (2005). Distributed coverage of unknown/unstructured environments by mobile sensor networks. In The Third MRS workshop.

Rekleitisy, I., Lee-Shuey, V., Newz, A. P., and Choset, H. (2004). Limited communication, multi-robot team based coverage. In IEEE International Conference on Robotics and Automation.

Shucker, B. and Bennett, J. (2005). Target tracking with distributed robotic macrosensors. In Military Communications Conference (MILCOM 2005), volume 4, pages 2617-2623.

Steels, L. (1990). Cooperation between distributed agents through self-organization. In Y.DeMazeau and J.P.Muller, editors, Decentralized A.I - Proc. first European Workshop on Modeling Autonomous Agents in Multi-Agents world, pages 175-196. Elsevier.

Stone, L. (1975). Theory of Optimal Search. Academic Press, New York.

Svennebring, J. and Koenig, S. (2004). Building terraincovering ant robots: A feasibility study. Autonomous Robots, 16(3):313-332.

Thayer, S., Dias, M., Digney, B., Stentz, A., Nabbe, B., and Hebert, M. (2000). Distributed robotic mapping of extreme environments. In Proceedings of SPIE, volume 4195 of Mobile Robots XV and Telemanipulator and Telepresence Technologies VII.

Vincent, P. and Rubin, I. (2004). A framework and analysis for cooperative search using uav swarms. In $A C M$ Simposium on applied computing.

Wagner, I., Altshuler, Y., Yanovski, V., and Bruckstein, A. (2008). Cooperative cleaners: A study in ant robotics. The International Journal of Robotics Research (IJRR), 27(1):127-151.

Wagner, I. and Bruckstein, A. (2001). From ants to a(ge)nts: A special issue on ant-robotics. Annals of Mathematics and Artificial Intelligence, Special Issue on Ant Robotics, 31(1-4):1-6.

Yamashita, A., Fukuchi, M., Ota, J., Arai, T., and Asama, H. (2000). Motion planning for cooperative transportation of a large object by multiple mobile robots in a $3 \mathrm{~d}$ environment. In In Proceedings of IEEE International Conference on Robotics and Automation, pages 3144-3151. 